\title{
Combination of long noncoding RNA MALATI and carcinoembryonic antigen for the diagnosis of malignant pleural effusion caused by lung cancer
}

This article was published in the following Dove Press journal: OncoTargets and Therapy

\author{
Wan-Wei Wang' \\ Xi-Lei Zhou' \\ Ying-Jian Song ${ }^{2}$ \\ Chang-Hua $\mathrm{Yu}^{\prime}$ \\ Wei-Guo Zhu' \\ Yu-Suo Tong' \\ 'Department of Radiation Oncology, \\ Huai'an First People's Hospital, \\ Nanjing Medical University, Huai'an, \\ Jiangsu, China; ${ }^{2}$ Department of \\ Respiratory Medicine, Huai'an First \\ People's Hospital, Nanjing Medical \\ University, Huai'an, Jiangsu, China
}

Correspondence: Yu-Suo Tong; Wei-Guo Zhu

Department of Radiation Oncology, Huai'an First People's Hospital, Nanjing Medical University, 6 Beijing Road West, Huai'an, Jiangsu 223300, China

Email tongyusuo@163.com; jshazwg@I26.com
Purpose: Long noncoding RNAs (lncRNAs) are present in body fluids, but their potential as tumor biomarkers has never been investigated in malignant pleural effusion (MPE) caused by lung cancer. The aim of this study was to assess the clinical significance of lncRNAs in pleural effusion, which could potentially serve as diagnostic and predictive markers for lung cancerassociated MPE (LC-MPE).

Patients and methods: RNAs from pleural effusion were extracted in 217 cases of LC-MPE and 132 cases of benign pleural effusion (BPE). Thirty-one lung cancer-associated lncRNAs were measured using quantitative real-time polymerase chain reaction (qRT-PCR). The level of carcinoembryonic antigen (CEA) was also determined. The receiver operating characteristic (ROC) curves and the area under the ROC curve (AUC) were established to evaluate the sensitivity and specificity of the identified lncRNAs and other biomarkers. The correlations between baseline pleural effusion lncRNAs expression and response to chemotherapy were also analyzed.

Results: Three lncRNAs (MALAT1, H19, and CUDR) were found to have potential as diagnostic markers in LC-MPE. The AUCs for MALAT1, H19, CUDR, and CEA were 0.891, 0.783, 0.824 , and 0.826, respectively. Using a logistic model, the combination of MALAT1 and CEA (AUC, 0.924 ) provided higher sensitivity and accuracy in predicting LC-MPE than CEA (AUC, 0.826) alone. Moreover, baseline MALAT1 expression in pleural fluid was inversely correlated with chemotherapy response in patients with LC-MPE.

Conclusion: Pleural effusion lncRNAs were effective in differentiating LC-MPE from BPE. The combination of MALATI and CEA was more effective for LC-MPE diagnosis.

Keywords: malignant pleural effusion, lncRNA, MALAT1, lung cancer, diagnosis

\section{Introduction}

Lung cancer remains the leading cause of cancer-related mortality globally, with almost one in five deaths attributable to it. ${ }^{1}$ Non-small cell lung cancer (NSCLC) compromises $>85 \%$ of all lung cancer cases. ${ }^{2}$ Most patients with NSCLC are diagnosed with advanced disease, and malignant pleural effusion (MPE) presents in $11 \%-32 \%$ of these patients. ${ }^{3}$ MPE can occur in patients with lung cancer of all histological types; however, it is particularly common in those with adenocarcinoma. ${ }^{4}$ The importance of accurately assessing MPE was emphasized by the seventh edition of the tumornode-metastasis classification for lung cancer, in which its status was reclassified as a stage IV disease. ${ }^{5}$ Currently, the differential diagnosis between benign pleural effusion (BPE) and lung cancer-associated MPE (LC-MPE) is extremely difficult, and only $50 \%-60 \%$ of LC-MPE cases can be diagnosed by cytological examination. ${ }^{6}$ Although thoracoscopic surgery and thoracotomy could improve the diagnostic power, they are 
too invasive for patients with a poor performance status, and many hospitals do not have these technologies, limiting their clinical application. ${ }^{7}$ Carcinoembryonic antigen (CEA) in pleural effusion is the most commonly used biomarker for diagnosing LC-MPE, but the sensitivity of detection is often unsatisfactory. ${ }^{8}$ Therefore, establishment of noninvasive biomarkers that could supplement CEA to improve the efficacy for diagnosing LC-MPE has important clinical implications.

Long noncoding RNAs (lncRNAs), which are commonly defined as transcripts longer than 200 nucleotides, play a critical role in the regulation of diverse cellular processes such as proliferation, cell cycle progression, cell growth and apoptosis, and cancer metastasis. ${ }^{9}$ Recent studies have reported that lncRNAs, which are involved in tumorigenesis and tumor progression, are present in various biological fluids including plasma, saliva, and urine. ${ }^{10-13}$ Although the mechanisms by which lncRNAs are released into body fluids remain largely unknown, several studies have indicated that they can be secreted from tumor cells. ${ }^{14}$ Thus, they could serve as biomarkers for cancer diagnosis. For example, Zhou et al reported that lncRNA H19 expression was significantly upregulated in the tumor tissue and plasma of gastric cancer patients and could be used to discriminate cancer patients from healthy controls. ${ }^{15}$ In addition, Tang et al demonstrated that the lncRNAs RP11-160H22.5, XLOC_014172, and LOC149086 were upregulated in the tissue and plasma of hepatocellular carcinoma patients compared with their levels in cancerfree controls, and could act as biomarkers for predicting the occurrence and metastasis of hepatocellular carcinoma. ${ }^{16}$ At present, although many lung cancer-associated lncRNAs have been found, their diagnostic or predictive value in pleural effusion has never been explored.

In the present study, 31 lncRNAs proven to have specificity for lung cancer in a previous study were chosen as candidate diagnostic markers. They were examined in pleural effusion, and their potential use as tumor markers for distinguishing LC-MPE from BPE was evaluated and compared with that of CEA in pleural effusion. The correlations between baseline lncRNAs expression in patients with LC-MPE and chemotherapy response were also investigated.

\section{Materials and methods Ethics statement}

The study was approved by the Research Ethics Committee, Huai'an First People's Hospital, Nanjing Medical University, and written informed consent was obtained from all patients.

\section{Clinical samples}

Three hundred and forty-nine patients with pleural effusion were recruited for this study from Huai'an First People's Hospital, Nanjing Medical University between January 2013 and December 2015. The presence of pleural effusion was demonstrated by a chest computed tomography scan. All patients underwent thoracentesis and pleural fluid cytology. For patients with an exudate of unknown origin, a second thoracentesis, pleural biopsy, or thoracoscopy was performed.

We divided the enrolled patients into two groups: LC-MPE $(n=217)$ and BPE $(n=132)$. LC-MPE was diagnosed by the presence of malignant cells in pleural fluid or pleural biopsy specimens, or if patients had disseminated malignancy and there was no alternative explanation for pleural effusion. All of the lung cancer patients were newly diagnosed and showed no evidence of another malignant tumor. The primary pulmonary lesion was classified as 68 squamous cell carcinomas, and 149 adenocarcinomas. Pleural effusions were diagnosed as benign based on the clinical context and the absence of malignant cells in at least two separate samples from the same patients. BPE samples were obtained from patients with no clinical or radiological evidence of malignancy prior to the study. Of the 132 patients with BPE, 50 were caused by tuberculous pleurisy, 26 by congestive cardiac failure, and 56 by parapneumonic effusion. A detailed description of the patients can be found in the Supplementary materials.

Clinical data including age, gender, and CEA are presented in Table 1 . The pleural effusion values of CEA were

Table I Clinical characteristics of patients

\begin{tabular}{|c|c|c|c|}
\hline Characteristics & $\begin{array}{l}\text { LC-MPE } \\
(n=2 \mid 7)\end{array}$ & $\begin{array}{l}\text { BPE } \\
(n=132)\end{array}$ & $p$-value \\
\hline Age (years) & & & 0.540 \\
\hline$\leq 55$ & 63 & 34 & \\
\hline$>55$ & 154 & 98 & \\
\hline Gender & & & 0.911 \\
\hline Male & 124 & 74 & \\
\hline Female & 93 & 58 & \\
\hline \multicolumn{4}{|l|}{ Pathological type } \\
\hline Adenocarcinoma & 149 & - & \\
\hline Squamous cell carcinoma & 68 & - & \\
\hline \multicolumn{4}{|l|}{ Diagnosis of BPE } \\
\hline Tuberculous pleurisy & - & 50 & \\
\hline Parapneumonic effusion & - & 56 & \\
\hline Congestive cardiac failure & - & 26 & \\
\hline CEA & & & $<0.001$ \\
\hline Positive (>5 ng/mL) & 122 & 9 & \\
\hline Negative ( $\leq 5 \mathrm{ng} / \mathrm{mL})$ & 95 & 123 & \\
\hline
\end{tabular}

Note: Statistical analysis, chi-square test.

Abbreviations: LC-MPE, lung cancer-associated malignant pleural effusion; BPE, benign pleural effusion; CEA, carcinoembryonic antigen. 
measured by electrochemiluminescence immunoassay using an analyzer (Hoffman-la-Roche Ltd, Basel, Switzerland).

Pleural fluid was collected from each patient prior to any cancer-directed therapy. Briefly, the puncture site was chosen by ultrasound. The patient was positioned to sit on the edge of the bed, leaning forward, with his arms resting on a bedside table. After routine disinfection and draping, the epidermis was anesthetized with $2 \%$ lidocaine. Then, the needle was inserted into the pleural cavity. Once the pleural fluid was obtained, the needle was not advanced any further, and $10 \mathrm{~mL}$ pleural effusion was aspirated from each patient. All pleural samples were transported to the laboratory within 30 minutes of collection. The samples were then centrifuged at $12,000 \mathrm{~g}$ for 10 minutes at $4^{\circ} \mathrm{C}$ in a refrigerated microfuge. The supernatant from each sample was transferred to an RNase-free tube and stored at $-80^{\circ} \mathrm{C}$ until total RNA extraction.

Of the 217 patients with LC-MPE, 162 cases received four cycles of cisplatin-based doublet chemotherapy, including cisplatin in combination with paclitaxel $(n=37)$, cisplatin plus docetaxel $(n=56)$, cisplatin plus pemetrexed $(n=34)$, and cisplatin plus vinorelbine $(\mathrm{n}=35)$.

\section{Clinical response evaluation}

After the baseline evaluation, chemotherapy response was assessed after completion of the second and fourth cycle of chemotherapy. The response to treatment was assessed basically according to the Response Evaluation Criteria in Solid Tumor. ${ }^{41}$

\section{RNA extraction}

Total RNA was isolated from pleural effusion samples using mirVana PARIS Kit (Ambion 1556; Thermo Fisher Scientific, Waltham, MA, USA) according to the manufacturer's protocol. In brief, all pleural effusion samples were thawed on ice, and $400 \mu \mathrm{L}$ of each sample was transferred to a tube containing an equal volume of $2 \times$ denaturing solution. Then, a volume of acid-phenol:chloroform was added equal to the total volume of the sample lysate plus the $2 \times$ denaturing solution. After centrifugation, the aqueous phase was recovered and transferred to a fresh tube. Subsequently, the lysate/ethanol mixture was passed through a filter cartridge, and the filter washed with wash solution. Finally, the RNA was eluted with $40 \mu \mathrm{L} 95^{\circ} \mathrm{C}$ nuclease-free water, and stored at $-80^{\circ} \mathrm{C}$. Eluted RNA from each sample was quantified by NanoDrop ND-1000 (Thermo Fisher Scientific), and the mean amount of total
RNA isolated from $400 \mu \mathrm{L}$ of pleural effusion was $220.5 \mathrm{ng}$ (range, 120.5-350.8 ng).

\section{qRT-PCR analysis of IncRNAs expression}

The concentrations of lncRNAs were quantified by quantitative real-time polymerase chain reaction (qRT-PCR) using SYBR ${ }^{\circledR}$ Premix Ex Taq ${ }^{\mathrm{TM}}$ II (Takara, Kyoto, Japan). The sequences of the qRT-PCR primers used in the present study are listed in Table S1. Briefly, a fixed volume of $5 \mu \mathrm{L}$ of total RNA elute from $40 \mu \mathrm{L}$ eluates was reverse transcribed in cDNA using PrimeScript $^{\mathrm{TM}}$ RT reagent kit with gDNA Eraser (Takara; RR047A) according to the manufacturer's instructions in a reaction volume of $20 \mu \mathrm{L}$. For the synthesis of cDNA, the reaction mixtures were incubated at $37^{\circ} \mathrm{C}$ for 15 minutes, followed by $85^{\circ} \mathrm{C}$ for 5 seconds, and then held at $4^{\circ} \mathrm{C}$. Then, $2 \mu \mathrm{L}$ of cDNA solution was amplified using $10 \mu \mathrm{L}$ of SYBR ${ }^{\circledR}$ Premix Ex TaqTM $(2 \times), 1.6 \mu \mathrm{L}$ of primers, $0.4 \mu \mathrm{L}$ of ROX Reference Dye II, and $6 \mu \mathrm{L}$ of nuclease-free water in a final volume of $20 \mu \mathrm{L}$. All qRT-PCR procedures were run on the ABI 7500 Real-Time PCR machine (Thermo Fisher Scientific) under the following conditions: $95^{\circ} \mathrm{C}$ for 30 seconds, followed by 40 cycles at $95^{\circ} \mathrm{C}$ for 5 seconds and $60^{\circ} \mathrm{C}$ for 34 seconds. All amplification reactions were examined in triplicate and the specificity of each qRT-PCR reaction was confirmed by melt curve analyses. To normalize the expression levels of lncRNAs, we used GAPDH as the endogenous control. The expression levels of lncRNAs were calculated from the following equation: $\Delta \mathrm{Ct}=$ mean $\mathrm{Ct}_{\text {IncRNAs }}-$ mean $\mathrm{Ct}_{\text {reference }}$; wherein lower $\Delta \mathrm{Ct}$ values indicate higher expression. The relative expression of lncRNAs was calculated by using $2^{-\Delta \Delta \mathrm{CT}}$ method with $G A P D H$ as internal control to normalize the data. $\mathrm{Ct}$ values $>40$ were considered negative.

\section{Statistics analysis}

The statistical significance of differences in lncRNA expression between groups was analyzed by Student's $t$-test or the Mann-Whitney test. The Shapiro-Wilk test was used to determine whether lncRNA expression followed a normal distribution. The association between the level of lncRNA in pleural effusion and chemotherapy response was determined by the chi-square test. Receiver operating characteristic (ROC) curves and the area under the ROC curve (AUC) were established to evaluate the sensitivity and specificity of the identified lncRNAs. The optimal cutoff value was calculated using the highest sum of sensitivity and specificity. Statistical analyses were performed using SPSS software (version 20.0; IBM Corporation, Armonk, NY, USA). All tests were twosided and a $p$-value $<0.05$ was considered significant. 


\section{Results}

\section{Patient characteristics}

Baseline characteristics of the study participants are shown in Table 1. In total, 132 effusions were diagnosed as benign and 217 were diagnosed as malignant. Of the 132 cases with BPE, there were 58 women (44\%), and the median age was 57 years (range, 35-74 years). Of the 217 patients with LC-MPE, there were 93 women (43\%), and the median age was 58 years (range, $32-78$ years). As shown in Table 1, there were no significant differences in age or gender between the LC-MPE and BPE groups.

Of the 217 patients with LC-MPE, pleural fluid cytology was positive in 83 patients (38\%) at the initial cytological analysis, and the remaining 134 patients were demonstrated by multiple thoracentesis $(n=27)$, pleural biopsy $(n=38)$, thoracoscopy $(n=8)$, and the presence of disseminated malignancy; there were no alternative explanations for pleural effusion ( $\mathrm{n}=61$ ). Among the 217 patients, elevated levels of CEA ( $>5 \mathrm{ng} / \mathrm{mL})$ were detected in $122(56 \%)$ patients.

\section{Lung cancer-associated IncRNAs are present in human pleural effusion samples}

Thirty-one lncRNAs were selected based on published studies that reported their differential expression in lung cancer patients (Table S1). To investigate whether these lncRNAs were present in human pleural effusion, all 31 candidate IncRNAs were then detected by qRT-PCR in pleural effusion from 10 LC-MPE and 10 age- and sex-matched BPE.

At present, there is no ideal housekeeping gene for the normalization of qRT-PCR data on pleural effusion IncRNAs. However, in our study, we found that the GAPDH level was stable in pleural effusion, and was not affected by age, sex, or pathology (Figure 1, Table 2). Thus, GAPDH was

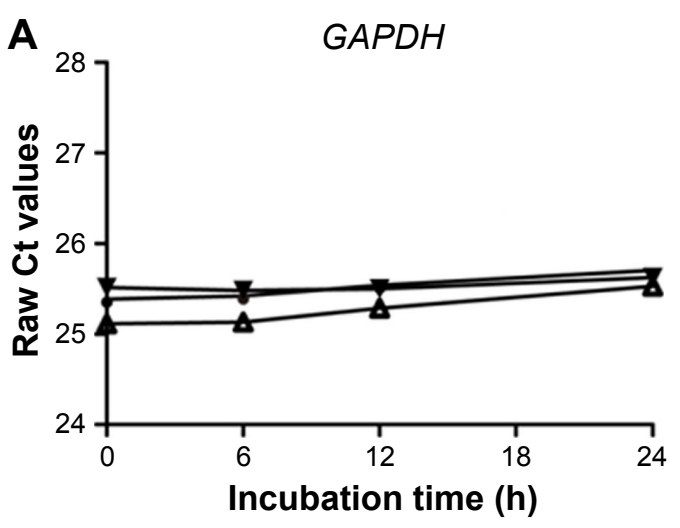

Sample 1

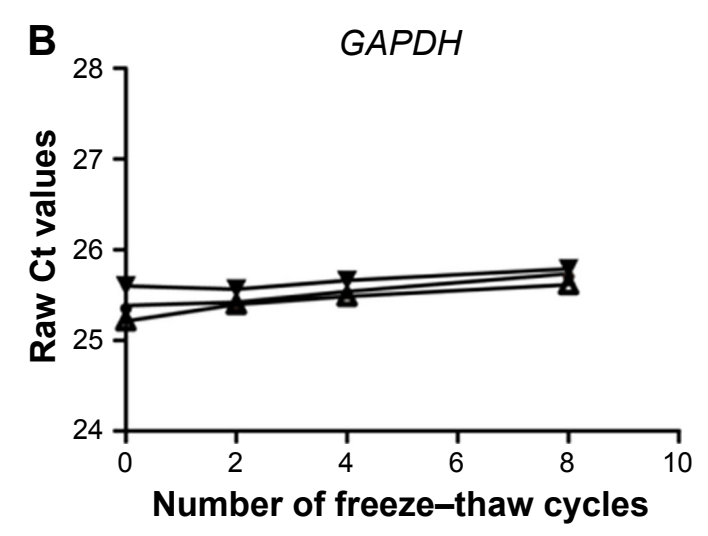

Sample 2

Sample 3

Figure I The stability of GAPDH in human pleural effusion.

Notes: Three pleural effusion samples were used in this experiment. Each pleural effusion sample was divided into two parts. One-half was incubated at room temperature for $0,6,12$, and 24 hours. For the second part, pleural effusion samples were subjected to multiple freeze-thaw cycles. GAPDH levels did not change significantly after prolonged incubation at room temperature $(\mathbf{A})$ or multiple freeze-thaw cycles $(\mathbf{B})$. Data presented as raw $\mathrm{Ct}$ values.
The expression levels of the remaining eight candidate lncRNAs were then measured by qRT-PCR in pleural effusions from all the subjects, including 217 LC-MPE and 132 BPE. As shown in Figure 2A-C, the levels of MALAT1, $H 19$, and $C U D R$ in pleural effusions were significantly higher in LC-MPE than in BPE, while the levels of PANDAR were significantly lower in LC-MPE than in the control group (Figure 2D). These results further suggest that lncRNAs can be utilized as a diagnostic tool for LC-MPE. However, the expression of SPRY4-IT1, ANRIL, NEAT-1, and PVT1 did not demonstrate any differences between LC-MPE and BPE ( $p>0.05$ ). Therefore, we selected MALAT1, H19, CUDR, and $P A N D A R$ as candidates for further analysis.

\section{Determination of the stability of lung cancer-associated IncRNAs in human pleural effusion} effusion, given that this is an important foundation for utility
We next assessed the stability of the four lncRNAs in pleural 
Table 2 Correlation between GAPDH level (raw Ct value) in human pleural effusion and clinicopathological factors of patients with BPE and LC-MPE

\begin{tabular}{llll}
\hline Variable & $\begin{array}{l}\text { No of } \\
\text { patients }(\%)\end{array}$ & $\begin{array}{l}\text { GAPDH level } \\
(\text { mean } \pm \text { SD) }\end{array}$ & p-value \\
\hline $\begin{array}{l}\text { Age (years) } \\
\quad<55\end{array}$ & $97(28)$ & $26.170 \pm 2.239$ & 0.360 \\
$\geq 55$ & $252(72)$ & $25.942 \pm 2.018$ & \\
$\begin{array}{l}\text { Gender } \\
\text { Male }\end{array}$ & $198(57)$ & $25.925 \pm 2.040$ & 0.409 \\
Female & $151(43)$ & $26.111 \pm 2.137$ & \\
$\begin{array}{l}\text { Pathological diagnosis } \\
\text { LC-MPE }\end{array}$ & $217(62)$ & $25.918 \pm 2.049$ & \\
BPE & $132(38)$ & $26.150 \pm 2.134$ & \\
\hline
\end{tabular}

Abbreviations: LC-MPE, lung cancer-associated malignant pleural effusion $\mathrm{BPE}$, benign pleural effusion; SD, standard deviation.

as tumor markers. The pleural effusion samples were treated under harsh conditions including extended storage, strong acid and base treatment, freeze-thaw cycles, and RNase digestion. A total of three pleural effusion samples were used in this part of the study, and each pleural effusion sample was divided into four parts. As shown in Figure 3A and B, the incubation of a pleural effusion sample at room temperature for up to 24 hours or subjecting it to treatment with a low- or high-pH solution had minimal influence on the expression of MALAT1, H19, $C U D R$, and PANDAR, as detected by qRT-PCR. Moreover, the four lncRNAs remained stable when the pleural effusion samples were treated with RNase A digestion or multiple freeze-thaw cycles (Figure 3C and D).

\section{Diagnostic utility of MALATI, HI 9, CUDR, PANDAR, and CEA for LC-MPE}

The diagnostic performance of MALAT1, H19, CUDR, and PANDAR for distinguishing LC-MPE from BPE was evaluated by ROC curve analysis. As shown in Figure 4A-D, the ROC curves of MALAT1, H19, and CUDR exhibited strong separation between LC-MPE and BPE, with AUCs of 0.891 (95\% CI: $0.854-0.928, p<0.001), 0.783$ (95\% CI: $0.730-0.836, p<0.001$ ), and 0.824 (95\% CI: $0.779-0.869$, $p<0.001)$, respectively, compared with CEA in pleural effusion with an AUC of 0.826 (95\% CI: 0.784-0.869, $p<0.001$ ). The cutoff values of MALAT1, H19, and CUDR were 6.975, 6.595 , and 6.005 , respectively. The sensitivity, specificity, and accuracy of the three lncRNAs for distinguishing LC-MPE from BPE are listed in Table 3. Among all the candidates, MALAT1 showed the highest sensitivity and specificity in discriminating LC-MPE from BPE. However, $P A N D A R$ in pleural effusion could not clearly discriminate LC-MPE from BPE (Figure 4E; AUC, 0.657, $p<0.001$ ).

The use of the three lncRNAs together provided an even more powerful diagnostic tool for differentiating LC-MPE from BPE. Using a logistic model, the AUC for the three lncRNAs combined increased to 0.962 (95\% CI: $0.942-0.982, p<0.001)$ with sensitivity of $91.7 \%$ and specificity of $89.4 \%$ (Figure $4 \mathrm{~F}$ ).

\section{Combination of MALATI and CEA for LC-MPE diagnosis}

One of the main aims of this study was to increase the diagnostic sensitivity of CEA. The combination of MALAT1 and CEA for distinguishing LC-MPE from BPE was analyzed by logistic regression analysis. As shown in Figure 5, this combination yielded an AUC of 0.924 (95\% CI: 0.893-0.954, $p<0.001)$, which was a significant improvement compared with those for CEA and MALAT1 alone. The sensitivity and specificity of this combination were $84.8 \%$ and $90.9 \%$, respectively (Table 3 ).

\section{Association of baseline IncRNA expressions in pleural effusion with chemotherapy response}

The measurement of biomarkers is not only an effective means of detecting tumors, but also an important way of predicting treatment response. In this study, 162 lung cancer patients received cisplatin-based chemotherapy. After four cycles of chemotherapy, complete response (CR), partial response (PR), stable disease, and progressive disease were
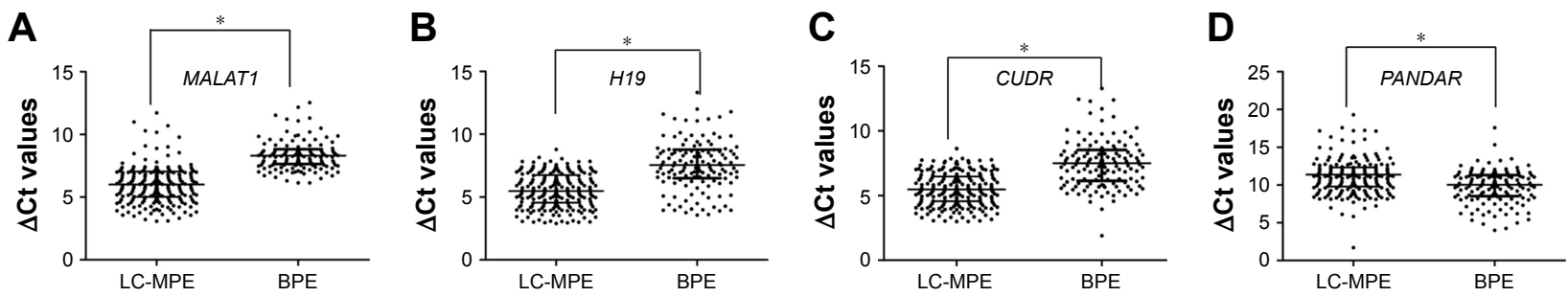

Figure 2 Comparison of pleural fluid levels of MALATI (A), HI9 (B), CUDR (C), and PANDAR (D) in LC-MPE and BPE.

Notes: $\triangle \mathrm{Ct}$ values were used to measure IncRNAs' expression, which was normalized by GAPDH expression, and a lower $\triangle \mathrm{Ct}$ value indicated higher expression. Horizontal bars indicated median and interquartile range. Statistical differences were analyzed using Mann-Whitney test. ${ }^{*} p<0.001$.

Abbreviations: LC-MPE, lung cancer-associated malignant pleural effusion; BPE, benign pleural effusion; IncRNAs, long noncoding RNAs. 
A

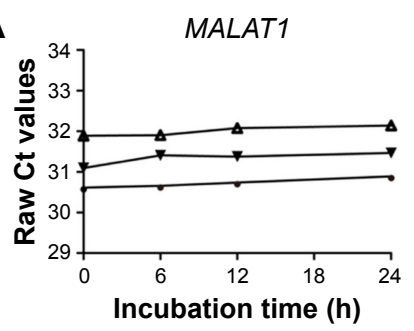

B

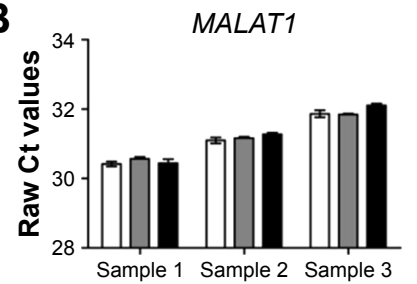

C

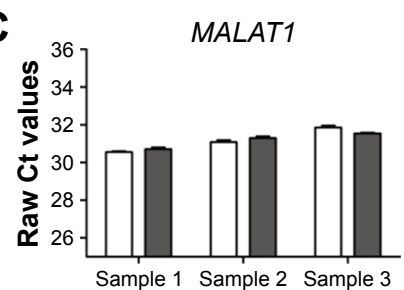

D

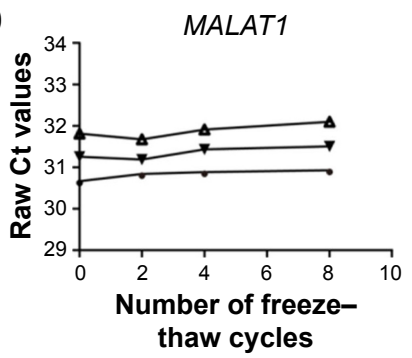

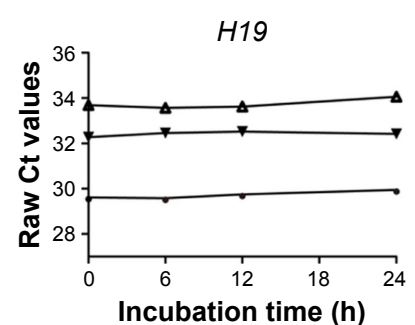
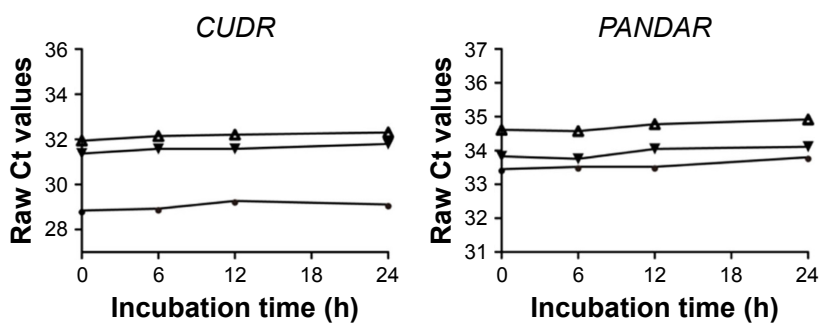

$\leftarrow$ Sample $1 \rightarrow$ Sample $2 \nrightarrow$ Sample 3
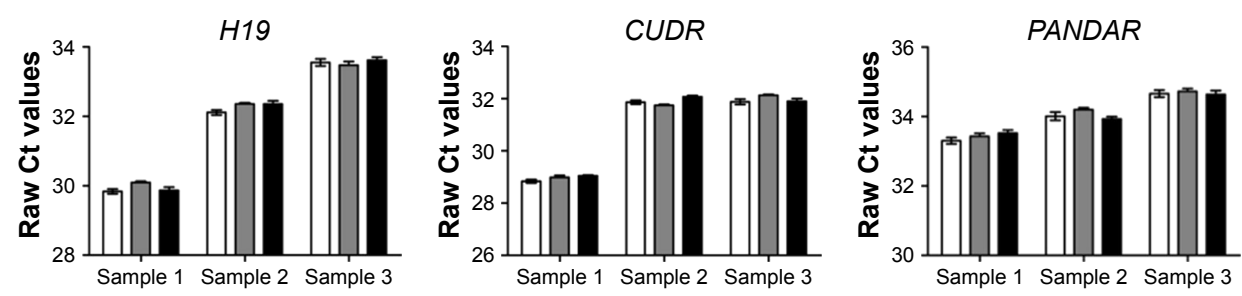

$\square$ No treatment $\square \mathrm{HCl}$ treatment $\square \mathrm{NaOH}$ treatment
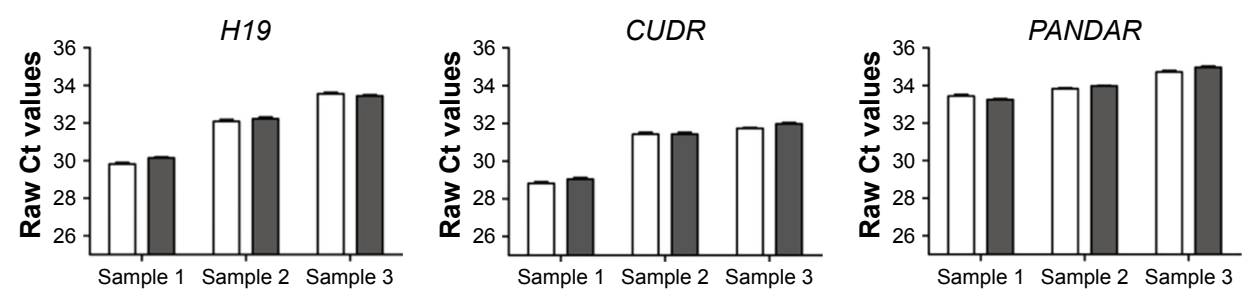

$\square$ No treatment $\square$ RNase A treatment
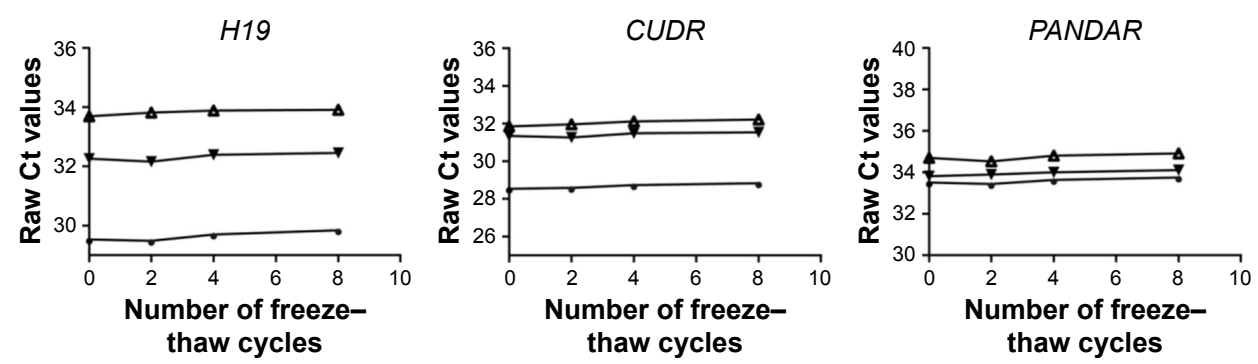

Sample $1 \rightarrow$ Sample $2 \star$ Sample 3

Figure 3 The stability of IncRNAs in human pleural effusion.

Notes: The expression of MALATI, HI9, CUDR, and PANDAR remained stable when the pleural effusion samples were subjected to extended room temperature incubation $(\mathbf{A})$, or treated for 3 hours in strong acid $(\mathrm{pH}=\mathrm{l})$ or base $(\mathrm{pH}=13)$ solution $(\mathbf{B}), \mathrm{p}>0.05$. The expressions of the four IncRNAs remained relatively stable when pleural fluids were treated with RNase A digestion (C) or multiple freeze-thaw cycles (D). Data presented as raw $\mathrm{Ct}$ values, $p>0.05$.

Abbreviation: IncRNAs, long noncoding RNAs.

observed in five patients ( $3 \%), 52$ patients $(32 \%), 71$ patients (44\%), and 34 patients (21\%), respectively. To investigate the association of baseline MALAT1 expression with chemotherapy response, the expression levels of MALAT1 in pleural effusion were categorized as high $(\mathrm{n}=81)$ and low $(\mathrm{n}=81)$ in relation to the median expression $(\Delta \mathrm{Ct}=6.155)$. The objective response rate $(\mathrm{CR}+\mathrm{PR})$ was significantly lower in patients with baseline MALAT1 $<6.155$ than in patients with $M A L A T 1 \geq 6.155$ ( $22 \%$ vs $49 \%, p<0.001$ ), demonstrating that MALAT1 in pleural fluid could be predictive of chemotherapy response in lung cancer before treatment. Unexpectedly, no significant correlations were observed between treatment response and clinicopathological parameters, such as age, gender, pathological type, and $H 19$ and CUDR expressions in pleural effusion ( $p>0.05$ ).

\section{Discussion}

To address several unanswered questions regarding the use of IncRNAs in pleural effusion biomarkers in LC-MPE, we focused on the pleural effusion levels of a set of 
A

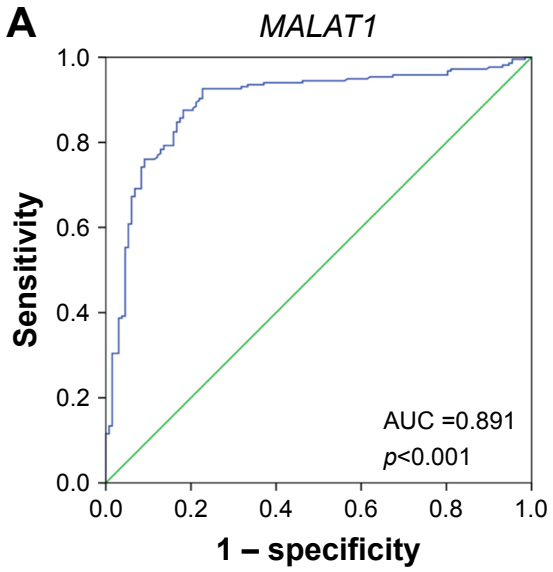

B

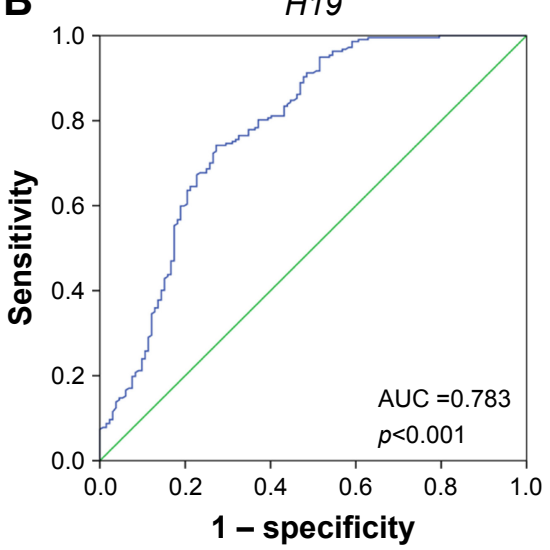

C
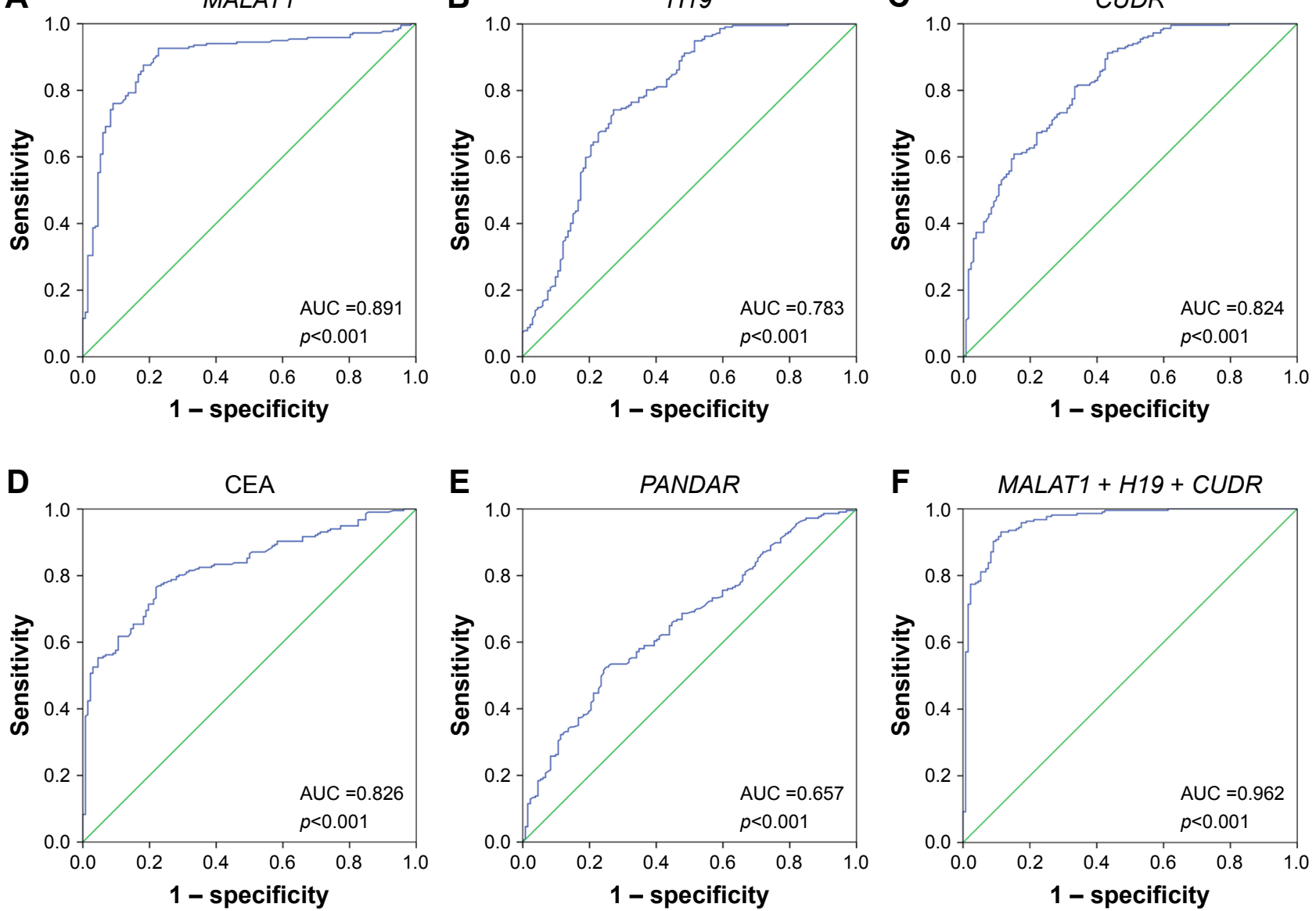

E

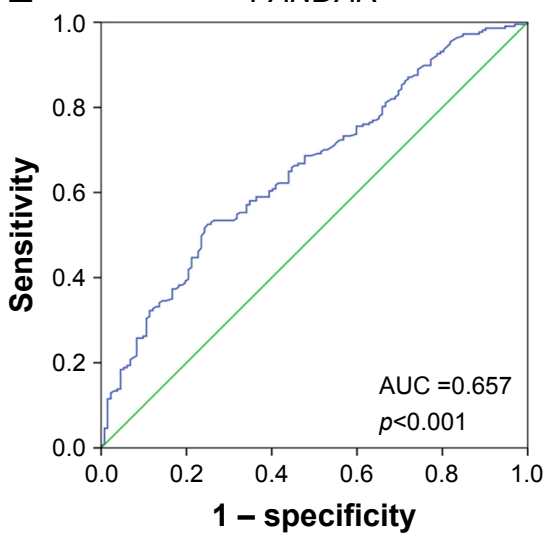

$\mathbf{F}$

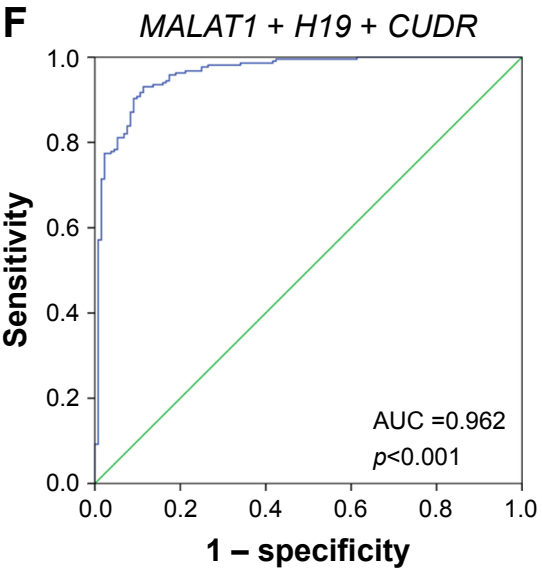

Figure 4 Evaluation of pleural fluid IncRNAs for the diagnosis of LC-MPE.

Notes: Receiver operating characteristics curves were drawn with the data of pleural fluid IncRNAs from 217 patients with LC-MPE and I 32 BPE. The values of AUC for MALATI (A), HI9 (B), CUDR (C), CEA (D), PANDAR (E), and the three IncRNAs combined (F) to differentiate LC-MPE from BPE were 0.89I, 0.783, 0.824, 0.826, 0.657, and 0.962 , respectively.

Abbreviations: LC-MPE, lung cancer-associated malignant pleural effusion; BPE, benign pleural effusion; IncRNAs, long noncoding RNAs; AUCs, area under the curves; CEA, carcinoembryonic antigen.

31 tumor-associated lncRNAs differently expressed between lung cancer tissues and adjacent normal tissues. The patient groups included patients diagnosed with LCMPE and BPE. We observed that three lncRNAs, MALAT1, $H 19$, and $C U D R$, could be clinically useful as biomarkers for LC-MPE diagnosis. Moreover, the combination of MALATl and CEA provided higher sensitivity and accuracy in predicting LC-MPE than CEA alone. In addition, our study also found that lung cancer patients with high relative expression levels of MALAT1 in the pleural fluid were correlated with chemotherapy resistance. To our knowledge, this is the first study wherein lncRNAs in pleural fluid have been shown to act as useful diagnostic and predictive biomarkers in LC-MPE.

Table 3 Performance of pleural effusion IncRNAs and CEA in the differential diagnosis of LC-MPE from BPE

\begin{tabular}{|c|c|c|c|c|c|c|}
\hline Tumor marker & $\begin{array}{l}\text { Sensitivity } \\
(95 \% \mathrm{Cl})\end{array}$ & $\begin{array}{l}\text { Specificity } \\
(95 \% \mathrm{Cl})\end{array}$ & $\begin{array}{l}\text { Accuracy } \\
(95 \% \text { CI) }\end{array}$ & $\begin{array}{l}\text { Youden } \\
\text { index (\%) }\end{array}$ & $\mathbf{L R}+$ & LR- \\
\hline CEA & $56.2 \%(49.53-63.28)$ & $93.9 \%$ (89.78-98.02) & $70.5 \%(65.60-75.4)$ & 50.1 & 9.21 & 0.47 \\
\hline MALATI & $74.2 \%(68.32-80.08)$ & $90.9 \%(86.00-95.80)$ & $80.5 \%(76.38-84.62)$ & 65.1 & 8.15 & 0.28 \\
\hline HI9 & $73.3 \%(67.42-79.18)$ & $72.7 \%$ (65.06-80.34) & $73.1 \%(68.40-77.80)$ & 46.0 & 2.68 & 0.37 \\
\hline CUDR & $65.9 \%(59.63-72.17)$ & $78.0 \%(70.95-85.05)$ & $70.5 \%(65.80-75.20)$ & 43.9 & 3.00 & 0.44 \\
\hline MALATI + HI9+CUDR & $91.7 \%$ (87.98-95.42) & $89.4 \%$ (84.II-94.69) & $90.8 \%(87.86-93.74)$ & 81.1 & 8.65 & 0.09 \\
\hline CEA + MALATI & $84.8 \%(80.10-89.50)$ & $90.9 \%(86.00-95.80)$ & $87.1 \%(83.57-90.63)$ & 75.7 & 9.32 & 0.17 \\
\hline
\end{tabular}

Abbreviations: LC-MPE, lung cancer-associated malignant pleural effusion; BPE, benign pleural effusion; CEA, carcinoembryonic antigen; Cl, confidence interval; LR+, positive likelihood ratio; LR-, negative likelihood ratio; IncRNAs, long noncoding RNAs. 


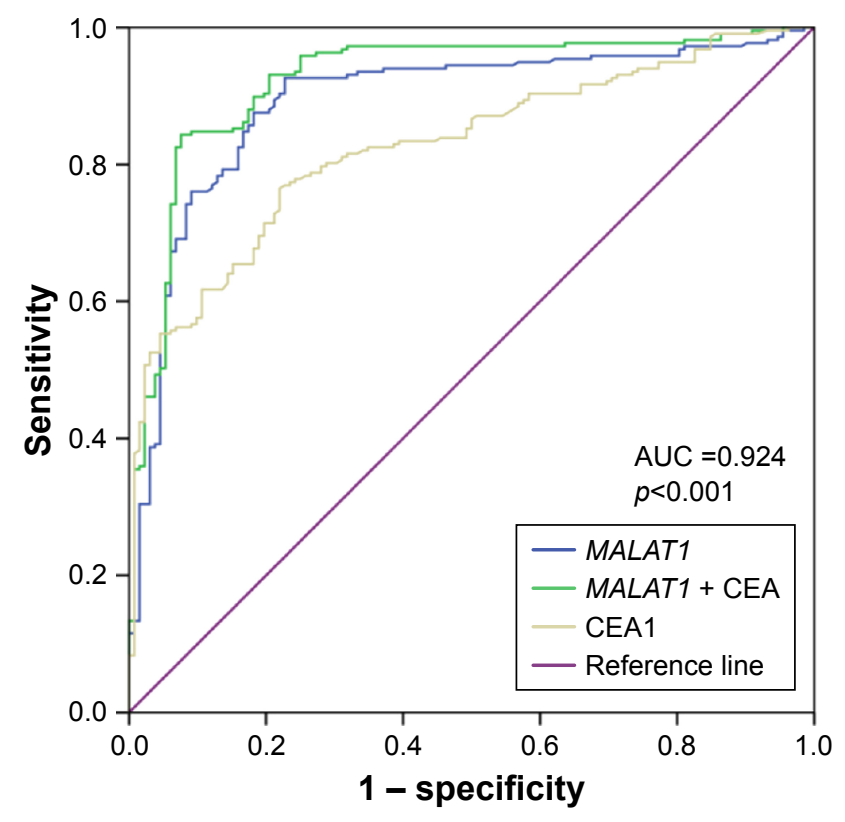

Figure 5 ROC curves to compare the ability of CEA, MALATI, and a combination of CEA and MALATI, to discriminate LC-MPE from BPE.

Notes: The AUC-ROC for distinguishing LC-MPE from BPE (CEA + MALATI, 0.924, $p<0.00$ I; MALATI, 0.89I, $p<0.0$; CEA, 0.826, $p<0.00$ I).

Abbreviations: LC-MPE, lung cancer-associated malignant pleural effusion; BPE, benign pleural effusion; AUC, area under the curve; ROC, receiver operating characteristic; CEA, carcinoembryonic antigen.

To date, no other tumor markers have been identified as being suitable to replace CEA in pleural fluid for LCMPE diagnosis. Elevated CEA in pleural fluid can predict LC-MPE with specificity of $>90 \%$, but the sensitivity is unsatisfactory. ${ }^{4}$ Recently, many biomarkers such as CA15-3, CA-125, CA-199, CYFRA 21-1, surviving and LUNX mRNA were assessed for LC-MPE diagnosis, but the sensitivities of these antigens were remarkably low (ranging from 0.376 to 0.625$).{ }^{17-19} \mathrm{CEA}$ in pleural fluid thus remains the most dependable of all biomarkers examined to date. Previous studies have indicated that lncRNAs are detectable in human fluids and revealed their potential utility as diagnostic markers in cancer. As early as 2002, de Kok et al demonstrated that IncRNA $P C A 3$ can be examined in the urine of patients with prostate cancer. ${ }^{20}$ The detection of IncRNA PCA3 in urine provided higher sensitivity and specificity for the diagnosis of prostate cancer than the widely used prostate-specificagent test. Our study examined lung cancer-related lncRNAs in pleural effusions from patients with LC-MPE and BPE. We found that the IncRNAs MALAT1, CUDR, and $H 19$ were significantly higher in LC-MPE than in BPE. MALAT1, also referred to as nuclear-enriched-abundant-transcript 2, was originally identified as a prognostic marker for metastasis and patient survival in gastric cancer and NSCLC. ${ }^{21,22}$ This lncRNA was widely expressed in normal human tissues, but was found to be overexpressed in multiple types of human malignancy, including hepatocellular carcinoma, breast, colon, lung, and prostate cancer, and it has served as a useful biomarker for the diagnosis of prostate and lung cancer. ${ }^{23}$ According to our results, lncRNA MALAT1 in pleural effusion might be an effective tumor marker to discriminate LC-MPE from BPE, and the diagnostic performance was better than the use of CEA. The combination of MALAT1 and CEA improved the diagnostic ability (AUC, 0.924); in particular, the sensitivity increased to $84.8 \%$.

qRT-PCR has become a versatile technique for examining gene expression. ${ }^{24}$ The use of reference genes as internal controls is the most common approach for improving the comparability of gene expression data. ${ }^{25}$ To produce reliable data on lncRNA expression levels, the selection of an appropriate reference gene is essential. A satisfactory reference gene should be present in all samples, and should also be stably expressed in all samples. Although several genes ( $\beta$-actin, GAPDH, HPRT1, RPLPO) have been used as endogenous controls in the normalization of tissue lncRNA expression, no endogenous control for normalizing lncRNAs in pleural effusion has been established. ${ }^{26}$ Our results demonstrated that $G A P D H$ levels remained stable after prolonged incubation at room temperature or repeated freezing and thawing, and were not affected by age, gender, or pathology. This is in accordance with the work of Shao et al, who used GAPDH for the normalization of lncRNAs in gastric juice samples of gastric cancer patients and normal controls. ${ }^{27}$

It has been reported that lncRNAs can be released into circulation from apoptotic and necrotic tumor cells and could be used to detect and monitor tumors. ${ }^{28}$ The 31 lncRNAs chosen for the present study were carefully selected with the hope of proving that they were secreted from or leaked out of lung cancer cells. However, with this selection process, the majority of IncRNAs with altered expression in lung cancer tissues did not show the same alteration in levels in pleural fluid in our study, which strongly suggested that the tumor tissues or the malignant tumor cells were not the sole origin of lncRNAs in pleural fluid. Fourteen of the 31 analyzed lncRNAs which were highly expressed in lung cancer tissues were undetected in pleural fluid caused by lung cancer. At present, the precise mechanisms of lncRNAs released in pleural fluid are still unclear. There are several possible explanations for this: the first is that lncRNAs found in pleural effusion might be released from blood cells or normal tissues. For example, Pritchard et al demonstrated that blood cells were the major contributors to the circulating miRNAs and that perturbations in blood cell counts and 
hemolysis can alter plasma miRNA concentrations by up to 50-fold. ${ }^{29}$ A second possible explanation is that lncRNAs found in pleural effusion are secreted and transferred from exosomes. Several studies have indicated that lncRNAs are abundant in exosomes and can be actively secreted from a variety of normal and tumor cells. ${ }^{30,31}$ Exosomes, which are automatically secreted, may transfer lncRNAs to body fluids. ${ }^{32,33}$ Another possible explanation is that the lncRNA levels in pleural fluid are correlated with immune responses. For example, some authors have speculated that the ncRNA signatures detected in patient plasma actually reflect the systemic responses to disease in the host microenvironment. ${ }^{34}$ Clearly, further analysis should be carried out to clarify the source of lncRNAs in pleural fluid and to understand their exact function in tumor progression.

After diagnosis, chemotherapy or targeted therapies are the main methods of treating patients with MPE caused by lung cancer. ${ }^{35,36}$ At present, the most commonly used chemotherapeutic regimen is cisplatin-based combination chemotherapy, consisting of cisplatin with a third-generation chemotherapy agent such as docetaxel, pemetrexed, or gemcitabine. ${ }^{37}$ However, NSCLC is poorly chemosensitive to most available agents with response rates ranging from $10 \%$ to $25 \% .{ }^{38}$ Thus, chemotherapy resistance is the main obstacle in the successful treatment of advanced NSCLC. In the present study, we found that baseline MALAT1 expression was inversely correlated with chemotherapy response in patients with LC-MPE. Several IncRNAs are known to be correlated with chemotherapysensitive phenotypes in cancers. We previously found that the lncRNA $A F A P 1-A S 1$ was significantly increased in the cisplatin-resistant KYSE30-R cell line compared with that in its parental cell line, and high expression of this lncRNA was significantly correlated with poor response to definitive chemoradiotherapy in patients with esophageal squamous cell carcinoma. ${ }^{39}$ Liu et al also demonstrated that the lncRNA $M E G 3$ was downregulated in lung cancer cells and partially regulated cisplatin resistance of tumor cells through the p53 signaling pathway. ${ }^{40}$ To the best of our knowledge, the correlation between MALAT1 expression and resistance to chemotherapy for lung cancer has not been analyzed.

The present study provides the first clinical evidence that lncRNAs in pleural effusion might have diagnostic potential for differentiating LC-MPE from BPE. However, this study has some limitations. First, the total number of patients included is relatively small. Second, we confirmed that MALAT1 expression predicted resistance to cisplatin-based chemotherapy in patients with LC-MPE. However, the exact mechanisms involved in this remained unclear, and no survival analysis to assess the prognostic value of MALATI was performed. In addition, several unknown pathological factors potentially affecting the expression of lncRNAs in pleural effusion might have influenced our results.

\section{Conclusion}

The lncRNAs MALAT1, H19, and CUDR in pleural effusion are valuable biomarkers for the diagnosis of LC-MPE. In particular, MALAT1 in pleural effusion shows higher diagnostic sensitivity and accuracy for LC-MPE, meaning that it is effective when used in combination with CEA for discriminating between LC-MPE and BPE.

\section{Acknowledgment}

This work was supported by a grant from the National Natural Science Foundation of China (grant no H1617/81602118).

\section{Author contributions}

WWW, WGZ, and YST conceived and designed the experiments and were responsible for writing the manuscript. XLZ was responsible for data analysis. CHY recruited the patients and collected their clinical data. All authors contributed toward data analysis, drafting and revising the paper and agree to be accountable for all aspects of the work.

\section{Disclosure}

The authors report no conflicts of interest in this work.

\section{References}

1. Minguet J, Smith KH, Bramlage P. Targeted therapies for treatment of non-small cell lung cancer - recent advances and future perspectives. Int J Cancer. 2016;138(11):2549-2561.

2. Ringner M, Jonsson G, Staaf J. Prognostic and chemotherapy predictive value of gene-expression phenotypes in primary lung adenocarcinoma. Clin Cancer Res. 2016;22(1):218-229.

3. Ryu JS, Ryu HJ, Lee SN, et al. Prognostic impact of minimal pleural effusion in non-small-cell lung cancer. J Clin Oncol. 2014;32(9):960-967.

4. Shin YM, Yun J, Lee OJ, et al. Diagnostic value of circulating extracellular miR-134, miR-185, and miR-22 levels in lung adenocarcinomaassociated malignant pleural effusion. Cancer Res Treat. 2014;46(2): 178-185

5. Han HS, Yun J, Lim SN, et al. Downregulation of cell-free miR-198 as a diagnostic biomarker for lung adenocarcinoma-associated malignant pleural effusion. Int J Cancer. 2013;133(3):645-652.

6. Bao QL, Li J, Sun W, Jiang HG, Zhu LR, Wang Y. Diagnostic utility of LUNX mRNA and VEGF mRNA in pleural fluid for differentiating benign from malignant origin. Jpn J Clin Oncol. 2014;44(12):1198-1205.

7. Tang Y, Xu L. Superiority and clinical significance of Lunx mRNA in the diagnosis of malignant pleural effusion caused by pulmonary carcinoma. $J$ Exp Clin Cancer Res. 2013;32:37.

8. Liang QL, Shi HZ, Qin XJ, Liang XD, Jiang J, Yang HB. Diagnostic accuracy of tumour markers for malignant pleural effusion: a metaanalysis. Thorax. 2008;63(1):35-41.

9. Qiu M, Xu Y, Wang J, et al. A novel lncRNA, LUADT1, promotes lung adenocarcinoma proliferation via the epigenetic suppression of $\mathrm{p} 27$ Cell Death Dis. 2015;6:e1858. 
10. Wu Y, Wang YQ, Weng WW, et al. A serum-circulating long noncoding RNA signature can discriminate between patients with clear cell renal cell carcinoma and healthy controls. Oncogenesis. 2016;5:e192.

11. XieZ, Chen X, Li J, et al. Salivary HOTAIR and PVT1 as novel biomarkers for early pancreatic cancer. Oncotarget. 2016;7(18):25408-25419.

12. Tong YS, Wang XW, Zhou XL, et al. Identification of the long noncoding RNA POU3F3 in plasma as a novel biomarker for diagnosis of ESCC. Mol Cancer. 2015;14:3.

13. Wang F, Ren S, Chen R, et al. Development and prospective multicenter evaluation of the long noncoding RNA MALAT-1 as a diagnostic urinary biomarker for prostate cancer. Oncotarget. 2014;5(22):11091-11102.

14. Reis EM, Verjovski-Almeida S. Perspectives of long non-coding RNAs in cancer diagnostics. Front Genet. 2012;3:32.

15. Zhou X, Yin C, Dang Y, Ye F, Zhang G. Identification of the long non-coding RNA H19 in plasma as a novel biomarker for diagnosis of gastric cancer. Sci Rep. 2015;5:11516.

16. Tang J, Jiang R, Deng L, Zhang X, Wang K, Sun B. Circulation long non-coding RNAs act as biomarkers for predicting tumorigenesis and metastasis in hepatocellular carcinoma. Oncotarget. 2015;6(6): 4505-4515.

17. Nguyen AH, Miller EJ, Wichman CS, Berim IG, Agrawal DK. Diagnostic value of tumor antigens in malignant pleural effusion: a metaanalysis. Transl Res. 2015;166(5):432-439.

18. Shitrit D, Zingerman B, Shitrit AB, Shlomi D, Kramer MR. Diagnostic value of CYFRA 21-1, CEA, CA 19-9, CA 15-3, and CA 125 assays in pleural effusions: analysis of 116 cases and review of the literature. Oncologist. 2005;10(7):501-507.

19. Wu YK, Chen KT, Kuo YB, Huang YS, Chan EC. Quantitative detection of survivin in malignant pleural effusion for the diagnosis and prognosis of lung cancer. Cancer Lett. 2009;273(2):331-335.

20. de Kok JB, Verhaegh GW, Roelofs RW, et al. DD3(PCA3), a very sensitive and specific marker to detect prostate tumors. Cancer Res. 2002; 62(9):2695-2698.

21. Gutschner T, Hämmerle M, Eissmann M, et al. The noncoding RNA MALAT1 is a critical regulator of the metastasis phenotype of lung cancer cells. Cancer Res. 2013;73(3):1180-1189.

22. Okugawa Y, Toiyama Y, Hur K, et al. Metastasis-associated long noncoding RNA drives gastric cancer development and promotes peritoneal metastasis. Carcinogenesis. 2014;35(12):2731-2739.

23. Qi P, Du X. The long non-coding RNAs, a new cancer diagnostic and therapeutic gold mine. Mod Pathol. 2012;26(2):155-165.

24. Taylor S, Wakem M, Dijkman G, Alsarraj M, Nguyen M. A practical approach to RT-qPCR-Publishing data that conform to the MIQE guidelines. Methods. 2010;50(4):S1-S5.

25. Davoren PA, McNeill RE, Lowery AJ, Kerin MJ, Miller N. Identification of suitable endogenous control genes for microRNA gene expression analysis in human breast cancer. BMC Mol Biol. 2008;9:76.

26. Weber DG, Johnen G, Casjens S, et al. Evaluation of long noncoding RNA MALAT1 as a candidate blood-based biomarker for the diagnosis of non-small cell lung cancer. BMC Res Notes. 2013;6:518.

27. Shao Y, Ye M, Jiang X, et al. Gastric juice long noncoding RNA used as a tumor marker for screening gastric cancer. Cancer. 2014;120(21): $3320-3328$.
28. Qi P, Zhou XY, Du X. Circulating long non-coding RNAs in cancer: current status and future perspectives. Mol Cancer. 2016;15(1):39.

29. Pritchard CC, Kroh E, Wood B, et al. Blood cell origin of circulating microRNAs: a cautionary note for cancer biomarker studies. Cancer Prev Res. 2011;5(3):492-497.

30. Berrondo C, Flax J, Kucherov V, et al. Expression of the long noncoding RNA HOTAIR correlates with disease progression in bladder cancer and is contained in bladder cancer patient urinary exosomes. PLoS One. 2016;11(1):e0147236.

31. Li Q, Shao Y, Zhang X, et al. Plasma long noncoding RNA protected by exosomes as a potential stable biomarker for gastric cancer. Tumour Biol. 2015;36(3):2007-2012.

32. Mohankumar S, Patel T. Extracellular vesicle long noncoding RNA as potential biomarkers of liver cancer. Brief Funct Genomics. 2016;15(3): 249-256.

33. Kogure T, Yan IK, Lin WL, Patel T. Extracellular vesicle-mediated transfer of a novel long noncoding RNA TUC339: a mechanism of intercellular signaling in human hepatocellular cancer. Genes Cancer. 2013;4(7-8):261-272.

34. Chen G, Wang J, Cui Q. Could circulating miRNAs contribute to cancer therapy. Trends Mol Med. 2013;19(2):71-73.

35. Su S, Li T, Lu B, et al. Three-dimensional radiation therapy to the primary tumor with concurrent chemotherapy in patients with stage IV non-small cell lung cancer: results of a multicenter phase 2 study from PPRA-RTOG, China. Int J Radiat Oncol Biol Phys. 2015;93(4): 769-777.

36. Sandler A, Gray R, Perry MC, et al. Paclitaxel-carboplatin alone or with bevacizumab for non-small-cell lung cancer. N Engl J Med. 2006; 355(24):2542-2550.

37. Yao Y, Yuan D, Liu H, Gu X, Song Y. Pretreatment neutrophil to lymphocyte ratio is associated with response to therapy and prognosis of advanced non-small cell lung cancer patients treated with first-line platinum-based chemotherapy. Cancer Immunol Immunother. 2013; 62(3):471-479.

38. Fortunato O, Boeri M, Moro M, et al. Mir-660 is downregulated in lung cancer patients and its replacement inhibits lung tumorigenesis by targeting MDM2-p53 interaction. Cell Death Dis. 2014;5:e1564.

39. Zhou XL, Wang WW, Zhu WG, et al. High expression of long noncoding RNA AFAP1-AS1 predicts chemoradioresistance and poor prognosis in patients with esophageal squamous cell carcinoma treated with definitive chemoradiotherapy. Mol Carcinog. 2016;55(12): 2095-2105.

40. Liu J, Wan L, Lu K, et al. The long noncoding RNA MEG3 contributes to cisplatin resistance of human lung adenocarcinoma. PLoS One. 2015; 10(5):e0114586.

41. Peng Y, Li Z, Zhang S, et al. Association of DNA base excision repair genes (OGG1, APE1 and XRCC1) polymorphisms with outcome to platinum-based chemotherapy in advanced nonsmall-cell lung cancer patients. Int J Cancer. 2014;135(11):2687-2696. 


\section{Supplementary materials}

Tuberculous pleurisy was diagnosed if acid-fast bacillus was identified in pleural effusion, or caseous granuloma was found in pleural biopsy specimens, or a high concentration of pleural fluid adenosine deaminase was examined $(>35 \mu / \mathrm{L})$. Parapneumonic effusion was diagnosed when there was acute febrile illness with pulmonary infiltrate and responsiveness to antibiotic treatment in patients with pleural effusion. A diagnosis of congestive cardiac failure was made by findings of an enlarged heart, pulmonary venous congestion on the radiograph, and with response to congestive cardiac failure treatment.

Table SI Primers sequences list

\begin{tabular}{|c|c|c|c|}
\hline Genes & Primers sequences $\left(5^{\prime}\right.$ to $\left.3^{\prime}\right)$ & Expression & Gene bank accession number \\
\hline \multirow[t]{2}{*}{ MALATI } & Forward: AACGCAGACGAAAATGGAAAGA & Up & NR_002819.10 \\
\hline & Reverse: CСТTCTAАСTTCTGCACCACCAGA & & \\
\hline \multirow[t]{2}{*}{ AFAPI-ASI } & Forward: TCGCTCAATGGAGTGACGGCA & Up & NR_026892.I \\
\hline & Reverse: CGGCTGAGACCGCTGAGAACTT & & \\
\hline \multirow[t]{2}{*}{ PVTI } & Forward: TGAGAACTGTCCTTACGTGACC & Up & NR_003367.2 \\
\hline & Reverse: AGAGCACCAAGACTGGCTCT & & \\
\hline \multirow[t]{2}{*}{ ANRIL } & Forward: TTGTGAAGCCCAAGTACTGC & Up & NR_047537.I \\
\hline & Reverse: TTCACTGTGGAGACGTTGGT & & \\
\hline \multirow[t]{2}{*}{ HNFIA-ASI } & Forward: TCAAGAAATGGTGGCTAT & Up & NR_024345.I \\
\hline & Reverse: GCTCTGAGACTGGCTGAA & & \\
\hline \multirow[t]{2}{*}{ PANDAR } & Forward: CCCAACAAACAAGGGGTGG & Down & NR_I09836.I \\
\hline & Reverse: GTGGCCAAAGGATCTGACGA & & \\
\hline \multirow[t]{2}{*}{ UCAI } & Forward: TTTATGCTTGAGCCTTGA & Up & NR_15379.3 \\
\hline & Reverse: CTTGCCTGAAATACTTGC & & \\
\hline \multirow[t]{2}{*}{ HOTAIR } & Forward: ATAGGCAAATGTCAGAGG GTT & Up & NR_0475I7.I \\
\hline & Reverse: ATTCTTAAATTGGGCTGGGTC & & \\
\hline \multirow[t]{2}{*}{ HI9 } & Forward: TGCTGCACTTTACAACCACTG & Up & NR_003958.2 \\
\hline & Reverse: ATGGTGTCTTTGATGTTGGGC & & \\
\hline \multirow[t]{2}{*}{ SPRY4-ITI } & Forward: AGCCACATAAATTCAGCAGA & Down & NR_13122I.I \\
\hline & Reverse: CGATGTAGTAGGATTCCTTTCA & & \\
\hline \multirow[t]{2}{*}{ TUGI } & Forward: TAGCAGTTCCCCAATCCTTG & Down & NR_002323.I \\
\hline & Reverse: CACAAATTCCCATCATTCCC & & \\
\hline \multirow[t]{2}{*}{ LINCOII33 } & Forward: GCTGTGGTGGAGAGAATGGA & Up & NR_038849.I \\
\hline & Reverse: CCCCAGCTTTCCAGATCCAAA & & \\
\hline \multirow[t]{2}{*}{ TUSC7 } & Forward: CTGAGCCAGCTTCACTGGAA & Up & NR_0I539I.I \\
\hline & Reverse: TCGTGCACATAGGCAGTGTT & & \\
\hline \multirow[t]{2}{*}{ ZXF2 } & Forward: CACCCAGGTCAGAGAAAGCA & Up & Not provided \\
\hline & Reverse: TGGAAGGGACACTAGAAGAAGAAT & & \\
\hline \multirow[t]{2}{*}{ LUADTI } & Forward: TTTCCGTTCAGCAAATCCACAC & Up & NR_I 32442.1 \\
\hline & Reverse: TTAGGTCCAGCACTGTTATCCA & & \\
\hline \multirow[t]{2}{*}{ CCAT2 } & Forward: CCCTGGTCAAATTGCTTAACCT & Up & NR_109834.I \\
\hline & Reverse: TTATTCGTCCCTCTGTTTTATGGAT & & \\
\hline \multirow[t]{2}{*}{ GAS5 } & Forward: CTTCTGGGCTCAAGTGATCCT & Down & NR_002578.2 \\
\hline & Reverse: TTGTGCCATGAGACTCCATCAG & & \\
\hline \multirow[t]{2}{*}{ MEG3 } & Forward: CTGCCCATCTACACCTCACG & Down & NR_002766.2 \\
\hline & Reverse: CTCTCCGCCGTCTGCGCTAGGGGCT & & \\
\hline \multirow[t]{2}{*}{ AGAP2-ASI } & Forward: TACCTTGACCTTGCTGCTCTC & Up & NR_027032.I \\
\hline & Reverse: TGTCCCTTAATGACCCCATCC & & \\
\hline \multirow[t]{2}{*}{ LINCOI 207} & Forward: CAGACACAGGCCATTCAGTC & Up & NR_038834.I \\
\hline & Reverse: CTTCTTCACCAGAAGCATTCC & & \\
\hline \multirow[t]{2}{*}{ CCATI } & Forward: TTTATGCTTGAGCCTTGA & Up & NR_I08049.I \\
\hline & Reverse: CTTGCCTGAAATACTTGC & & \\
\hline \multirow[t]{2}{*}{ GAS6-ASI } & Forward: GTGGGTACTGCATTCCTACCG & Down & NR_044995.2 \\
\hline & Reverse: СТСТССТСTGATGGCAGGAC & & \\
\hline \multirow[t]{2}{*}{ MVIH } & Forward: GAGACAGGATTTAGCCGTGTTG & Up & Not provided \\
\hline & Reverse: AGCACTTTGGAAGGCTTAGACA & & \\
\hline
\end{tabular}


Table SI (Continued)

\begin{tabular}{|c|c|c|c|}
\hline Genes & Primers sequences $\left(5^{\prime}\right.$ to $\left.3^{\prime}\right)$ & Expression & Gene bank accession number \\
\hline \multirow[t]{2}{*}{ IncRNA-LET } & Forward: GTTGTTGTTGCATTGGGGT & Down & NR_I03844.I \\
\hline & Reverse: AAGATGGAGAGTGGAGCCT & & \\
\hline \multirow[t]{2}{*}{ BANCR } & Forward: ACAGGACTCCATGGCAAACG & Down & NR_04767I.2 \\
\hline & Reverse: ATGAAGAAAGCCTGGTGCAGT & & \\
\hline \multirow[t]{2}{*}{ PCATI } & Forward: TAGAGCCTTGAAGATGAG & Up & NR_045262.I \\
\hline & Reverse: TCGTGTAGTTGTAAGATGA & & \\
\hline \multirow[t]{2}{*}{ CUDR } & Forward: GCACCCTAGACCCGAAA & $U_{p}$ & NR_0I5379.3 \\
\hline & Reverse: GCCACCTGGACGGATAT & & \\
\hline \multirow[t]{2}{*}{ DLX6-ASI } & Forward: AGTTTCTCTCTAGATTGCCTT & Up & NR_0I5448.I \\
\hline & Reverse: GACATGTTAGTGCCCTT & & \\
\hline \multirow[t]{2}{*}{ NEAT-I } & Forward: TGGCTAGCTCAGGGCTTCAG & Up & NR_028272.I \\
\hline & Reverse: TCTCCTTGCCAAGCTTCCTT & & \\
\hline \multirow[t]{2}{*}{ SOX2ot } & Forward: TTCGGAAAAGTGGCCATCCA & Up & NR_004053.3 \\
\hline & Reverse: TCACCCACATCGCCTTACAC & & \\
\hline
\end{tabular}

Abbreviation: IncRNA, long noncoding RNA.

\section{Publish your work in this journal}

OncoTargets and Therapy is an international, peer-reviewed, open access journal focusing on the pathological basis of all cancers, potential targets for therapy and treatment protocols employed to improve the management of cancer patients. The journal also focuses on the impact of management programs and new therapeutic agents and protocols on patient perspectives such as quality of life, adherence and satisfaction. The manuscript management system is completely online and includes a very quick and fair peer-review system, which is all easy to use. Visit http://www.dovepress.com/testimonials.php to read real quotes from published authors. 